Article

\title{
Molecular Analysis of the Interaction of the Snake Venom Rhodocytin with the Platelet Receptor CLEC-2
}

\author{
Aleksandra A. Watson ${ }^{1}$ and Christopher A. O'Callaghan ${ }^{2, *}$ \\ 1 Department of Biochemistry, University of Cambridge/ 80 Tennis Court Road, Cambridge, \\ CB2 1GA, UK; E-Mail: aaw36@ cam.ac.uk \\ 2 Henry Wellcome Building for Molecular Physiology, University of Oxford/ Roosevelt Drive, \\ Oxford, OX3 7BN, UK \\ * Author to whom correspondence should be addressed; E-Mail: chris.ocallaghan@ndm.ox.ac.uk; \\ Tel.: +44-1865-287789; Fax: +44-1865-287787.
}

Received: 6 July 2011; in revised from: 21 July 2011 / Accepted: 8 August 2011 /

Published: 10 August 2011

\begin{abstract}
The Malayan pit viper, Calloselasma rhodostoma, produces a potent venom toxin, rhodocytin (aggretin) which causes platelet aggregation. Rhodocytin is a ligand for the receptor CLEC-2 on the surface of platelets. The interaction of these two molecules initiates a signaling pathway which results in platelet activation and aggregation. We have previously solved the crystal structures of CLEC-2 and of rhodocytin, and have proposed models by which tetrameric rhodocytin may interact with either two monomers of CLEC-2, or with one or two copies of dimeric CLEC-2. In the current study we use a range of approaches to analyze the molecular interfaces and dynamics involved in the models of the interaction of rhodocytin with either one or two copies of dimeric CLEC-2, and their implications for clustering of CLEC-2 on the platelet surface.
\end{abstract}

Keywords: rhodocytin; CLEC-2; platelets; thrombosis

\section{Introduction}

A range of snake toxins have evolved to influence blood clotting and platelet aggregation [1]. Various C-type lectin-like proteins influence platelet aggregation through interactions with receptors on the surface of platelets; a number of these venom proteins are heterodimeric [2,3]. Platelet activation and aggregation can be triggered by various well defined receptors on the surface of 
platelets. Under typical physiological conditions, GPIb-V-IX and $\alpha \operatorname{IIb} \beta 3$ integrin interact with von Willebrand factor when the latter has become attached to extracellular collagen. Collagen itself interacts with the immunoglobulin superfamily receptor GPVI and the integrin $\alpha 2 \beta 1[4,5]$. There is great biomedical interest in the discovery of novel platelet-activating receptors and in this respect the identification of the targets on platelets for snake venom proteins has been an important objective.

The Malayan pit viper Calloselasma rhodostoma is a major cause of snakebite morbidity in large parts of Southeast Asia and effects on platelet function are key consequences of envenomation [6]. The pit viper produces a venom protein, rhodocytin (aggretin) which was purified and shown to cause powerful platelet activation and aggregation [7,8]. The molecular cloning and sequence analysis of rhodocytin demonstrated that the two subunits, designated alpha and beta, each have characteristics of the C-type lectin-like family [9]. Rhodocytin has been shown to be a ligand for CLEC-2, a recently identified receptor on the surface of platelets [10]. Binding of rhodocytin to CLEC-2 triggers a potent platelet signaling pathway [10,11]. CLEC-2 contains a single YXXL motif in its cytoplasmic tail. Rhodocytin binding leads to tyrosine phosphorylation in this cytoplasmic tail of CLEC-2, which promotes the binding of spleen tyrosine kinase (Syk), subsequent activation of PLC $\gamma 2$ and platelet activation and aggregation [10]. CLEC-2 is encoded in a genomic cluster with related C-type lectin-like molecules, some of which have immunological roles, as exemplified by NKG2D [12-17]. CLEC-2 was first identified as a receptor on platelets for rhodocytin and may also play a role in lymphatic development $[10,18]$. We and others have shown that podoplanin is an endogenous ligand for CLEC-2 [16,19]. Podoplanin is expressed on the luminal aspect of lymphatic endothelial cells and in a range of tissues including kidney, heart, lung and many tumours [20]. The biological importance of CLEC-2 and podoplanin is indicated by observations that genetic knockouts of either molecule are lethal during embryonic development [21-28].

The mechanism whereby rhodocytin triggers platelet aggregation is of great interest because a detailed knowledge of CLEC-2-mediated platelet activation could be of value in understanding and preventing platelet aggregation in thrombotic coronary and cerebral vascular disease, which are major causes of disability and death worldwide. Furthermore, there is a strong need to understand the rhodocytin-CLEC-2 interaction in its own right as snake envenomation affects over 2.5 million humans per year, causing more than 100,000 deaths [29]. We have solved the structure of the C-type lectin-like domain of CLEC-2 and have used mutagenesis to characterise the interaction with rhodocytin [30,31]. The binding affinity of CLEC-2 with rhodocytin and podoplanin has been measured using surface plasmon resonance (Biacore technology) [16,30,32]. We found the affinity of the interaction of rhodocytin with monomeric CLEC-2 to be $1.01 \pm 0.20 \mu \mathrm{M}$, as compared with $24.5 \pm 3.7 \mu \mathrm{M}$ and $4.1 \pm 0.2 \mu \mathrm{M}$ for the interactions of podoplanin with monomeric and dimeric CLEC-2 respectively [16,30,32].

To further explore the association between CLEC-2 and rhodocytin, we have also solved the crystal structure of rhodocytin, and discovered that it assembles as a non-disulfide linked $(\alpha \beta)_{2}$ tetramer [33]. Rhodocytin is the first snake venom or other C-type lectin-like protein reported to adopt this configuration. We proposed that the rhodocytin tetramer might induce clustering of CLEC-2 molecules on the platelet surface and that this could play a key role in triggering signaling to platelet activation. We have previously generated three models of the rhodocytin-CLEC-2 interaction, whereby tetrameric rhodocytin may promote clustering of CLEC-2 by interacting with two copies of monomeric CLEC-2, 
one copy of dimeric CLEC-2, or two copies of dimeric CLEC-2. We have since used a range of cellular, biochemical and biophysical techniques to demonstrate that CLEC-2 exists as a non-disulfidelinked homodimer [32]. Although Syk generally interacts with two YXXL motifs on a single polypeptide chain, there is evidence to indicate that it can interact with two YXXL motifs, each from a different CLEC-2 chain, consistent with CLEC-2 functioning in a dimeric manner in its interaction with Syk [34]. These observations preclude the first of our original models of the rhodocytin-CLEC-2 interaction, wherein two copies of monomeric CLEC-2 bind to tetrameric rhodocytin. In the current study, we analyse and discuss the interactions involved in the two other models of the interaction, where rhodocytin may bind either one or two copies of dimeric CLEC-2, and discuss the implications for clustering of CLEC-2 on the platelet surface.

\section{Materials and Methods}

\subsection{Protein Interfaces, Surfaces and Assemblies}

Algorithms implemented by PISA (Protein interfaces, surfaces and assemblies version 1.2) were used to explore the macromolecular protein interfaces of the model complexes of rhodocytin plus either one, or two copies of model dimeric CLEC-2 [35]. These models were generated as previously reported [33]. These calculations included the structural and chemical properties of macromolecular surfaces and interfaces, the accessible/buried surface area, the free energy of dissociation, and the presence or absence of salt bridges and disulfide bonds. The protein database archive (PDB) was searched for particular interfaces formed by structural homologs, and the PISA database was explored to compare results for multimeric state, symmetry number, space group, accessible/buried surface area, free energy of dissociation, presence/absence of salt bridges and disulfide bonds, homomeric type, and ligands. Structures, interfaces and assemblies were visualised for analysis using Rastop and Jmol (an open-source Java viewer for chemical structures in 3D. http://www.jmol.org/).

\subsection{Molecular Dynamics}

The potential modes of motion of the different models of the rhodocytin-CLEC-2 complex were examined and analysed using the Dynamite package [36]. From the input three dimensional structure, an ensemble of structures was generated, and subsequently analysed to predict which elements move together and the relevant vectors of these motions. The ensemble was generated using Concoord, and the analysis was performed using Gromacs. In essence, Concoord is used to identify all interatomic interactions in the structure which is input [37]. The likely strength of these interactions is analysed and so the potential freedom of the interacting species is modeled to within appropriate bounds. Following this, new variant structures are established that fulfill the limitations of these modeled bounds. These dynamic analyses were represented graphically using Visual Molecular Dynamics (VMD) [38]. 


\section{Results and Discussion}

\subsection{The Interaction Surfaces of the Rhodocytin-CLEC-2 Interaction}

There are two alpha and two beta subunits of rhodocytin per tetrameric unit. The most basic model of the interaction of rhodocytin with CLEC-2 is that in which one molecule of dimeric CLEC-2 is complexed with one molecule of rhodocytin, the rhodocytin being in the form of the non-disulfide linked $(\alpha \beta)_{2}$ tetramer that was identified by crystallography [33]. In this complex, there are two hydrogen bonds and one salt bridge involved in the interaction between dimeric CLEC-2 and one beta subunit of rhodocytin (Table 1). However, in this model, the other beta subunit of the tetramer does not bind to the CLEC-2 dimer (Table 1). In addition to the interactions with the beta subunit of rhodocytin, dimeric CLEC-2 interacts with one alpha subunit of rhodocytin through five hydrogen bonds and three salt bridges (Table 1). In contrast to the beta subunits, where only one interacts with CLEC-2, this second alpha subunit does interact with the CLEC-2 dimer in this model, but does not form any ionic bonds with it (Table 1). It is likely that this interaction with the second alpha subunit is mediated by van der Waals forces.

The more complex model of the rhodocytin-CLEC-2 interaction involves two copies of dimeric CLEC-2 bound to one molecule of the non-disulfide linked $(\alpha \beta)_{2}$ tetrameric form of rhodocytin. It is important to note that this model is distinct from that described above, and has been generated independently in a way that has not been influenced by the first model. In this complex, four hydrogen bonds and one salt bridge are involved in the interaction between each molecule of dimeric CLEC-2 and each rhodocytin beta subunit (Table 2). In addition, the interaction between each alpha subunit of rhodocytin and each molecule of dimeric CLEC-2 involves a further two hydrogen bonds and one salt bridge (Table 2). Clearly, this model in which two CLEC-2 dimers associate with tetrameric rhodocytin involves more numerous and favourable ionic interactions and hydrogen bonds (a total of sixteen, as opposed to eleven interactions per tetramer of rhodocytin) in addition to van der Waals contacts and would therefore be a more likely model (Figure 1). Interestingly, residue K150 of CLEC-2, which we identified as being important for its interaction with rhodocytin using mutagenesis and surface plasmon resonance, is also involved in the interface in this model of the complex [30].

There are 246 bound water molecules present in the crystal structure of monomeric CLEC-2 and 45 water molecules present in the crystal structure of rhodocytin. Therefore, upon dimerization of CLEC-2 and complexing of this dimeric CLEC-2 with tetrameric rhodocytin, there will be considerable liberation of the water molecules which solvate the unbound molecules. In our model where one copy of dimeric CLEC-2 binds tetrameric rhodocytin, the alpha subunits and the one beta subunit involved in the complex experience gains in the free energy of solvation of $1.5,2.5$ and $0.2 \mathrm{kcal} / \mathrm{mol}$ respectively (total $4.2 \mathrm{kcal} / \mathrm{mol}$ ). However, in our more complex model in which two CLEC-2 dimers bind to one tetramer of rhodocytin, the solvation energy gains for each CLEC-2 dimer interaction are 0.2 and $0.6 \mathrm{kcal} / \mathrm{mol}$ for the alpha subunits, and 2.2 and $0.7 \mathrm{kcal}$ for the two beta subunits (total $7.4 \mathrm{kcal} / \mathrm{mol}$ ). There is, therefore, a more appreciable gain in the free energy of solvation upon formation of the complex of two CLEC-2 dimers rather than one with tetrameric rhodocytin, which again indicates that this is a more plausible model. 
Table 1. Contact information and interfacing residues involved in the model interaction of one copy of dimeric CLEC-2 with tetrameric rhodocytin. Key: $\mathrm{H}=$ residues making hydrogen bonds, $\mathrm{S}=$ residues making a salt bridge, ASA = accessible surface area $\left(\AA^{2}\right)$, BSA $=$ buried surface area $\left(\AA^{2}\right), \Delta \mathrm{iG}=$ solvation energy effect $(\mathrm{kcal} / \mathrm{mol}),||||=$ buried area percentage, one bar per $10 \%$.

\begin{tabular}{|c|c|c|c|c|}
\hline & \multicolumn{2}{|c|}{ Rhodocytin alpha chain \#1 } & \multicolumn{2}{|c|}{$\begin{array}{l}\text { CLEC-2 } \\
\end{array}$} \\
\hline $\begin{array}{c}\text { Number of atoms } \\
\text { interface } \\
\text { surface } \\
\text { total } \\
\end{array}$ & $\begin{array}{r}17 \\
715 \\
1121 \\
\end{array}$ & $\begin{array}{l}(1.5 \%) \\
(63.8 \%) \\
(100.0 \%) \\
\end{array}$ & $\begin{array}{c}18 \\
1129 \\
2085 \\
\end{array}$ & $\begin{array}{l}(0.9 \%) \\
(54.1 \%) \\
(100.0 \%)\end{array}$ \\
\hline $\begin{array}{l}\text { Number of residues } \\
\text { interface } \\
\text { surface } \\
\text { total }\end{array}$ & $\begin{array}{c}8 \\
123 \\
133\end{array}$ & $\begin{array}{l}(6.0 \%) \\
(92.5 \%) \\
(100.0 \%)\end{array}$ & $\begin{array}{c}5 \\
222 \\
242\end{array}$ & $\begin{array}{l}(2.1 \%) \\
(91.7 \%) \\
(100.0 \%)\end{array}$ \\
\hline $\begin{array}{c}\text { Solvent-accessible area, } \tilde{\AA}^{2} \\
\text { interface } \\
\text { total }\end{array}$ & $\begin{array}{c}150.2 \\
8404.1\end{array}$ & $\begin{array}{c}(1.8 \%) \\
(100.0 \%)\end{array}$ & $\begin{array}{r}164.3 \\
12294.5 \\
\end{array}$ & $\begin{array}{c}(1.3 \%) \\
(100.0 \%)\end{array}$ \\
\hline $\begin{array}{l}\text { Solvation energy, kcal/mol } \\
\text { isolated structure } \\
\text { gain at complexation } \\
\text { average gain } \\
P \text {-value }\end{array}$ & $\begin{array}{l}-94.6 \\
-1.5 \\
-0.6 \\
0.229\end{array}$ & $\begin{array}{c}(100.0 \%) \\
(1.5 \%) \\
(0.6 \%)\end{array}$ & $\begin{array}{l}-222.4 \\
-0.1 \\
-0.1 \\
0.543\end{array}$ & $\begin{array}{c}(100.0 \%) \\
(0.0 \%) \\
(0.0 \%)\end{array}$ \\
\hline & \multicolumn{2}{|c|}{ Rhodocytin alpha chain \#2 } & \multicolumn{2}{|c|}{ CLEC-2 } \\
\hline $\begin{array}{c}\text { Number of atoms } \\
\text { interface } \\
\text { surface } \\
\text { total }\end{array}$ & $\begin{array}{c}52 \\
713 \\
1121\end{array}$ & $\begin{array}{l}(4.6 \%) \\
(63.6 \%) \\
(100.0 \%)\end{array}$ & $\begin{array}{c}68 \\
1129 \\
2085\end{array}$ & $\begin{array}{l}(3.3 \%) \\
(54.1 \%) \\
(100.0 \%)\end{array}$ \\
\hline $\begin{array}{c}\text { Number of residues } \\
\text { interface } \\
\text { surface } \\
\text { total }\end{array}$ & $\begin{array}{l}15 \\
123 \\
133\end{array}$ & $\begin{array}{l}(11.3 \%) \\
(92.5 \%) \\
(100.0 \%)\end{array}$ & $\begin{array}{l}22 \\
222 \\
242\end{array}$ & $\begin{array}{l}(9.1 \%) \\
(91.7 \%) \\
(100.0 \%)\end{array}$ \\
\hline $\begin{array}{l}\text { Solvent-accessible area, } \AA^{2} \\
\text { interface } \\
\text { total }\end{array}$ & $\begin{array}{c}587.3 \\
8416.9\end{array}$ & $\begin{array}{c}(7.0 \%) \\
(100.0 \%)\end{array}$ & $\begin{array}{c}537.5 \\
12294.5\end{array}$ & $\begin{array}{c}(4.4 \%) \\
(100.0 \%)\end{array}$ \\
\hline $\begin{array}{l}\text { Solvation energy, kcal/mol } \\
\text { isolated structure } \\
\text { gain at complexation } \\
\text { average gain } \\
P \text {-value }\end{array}$ & $\begin{array}{l}-94.8 \\
-2.5 \\
-1.7 \\
0.373\end{array}$ & $\begin{array}{l}(100.0 \%) \\
(2.6 \%) \\
(1.8 \%)\end{array}$ & $\begin{array}{l}-222.4 \\
2.8 \\
-0.3 \\
0.895\end{array}$ & $\begin{array}{c}(100.0 \%) \\
(-1.3 \%) \\
(0.2 \%)\end{array}$ \\
\hline
\end{tabular}

\begin{tabular}{|c|c|c|c|c|}
\hline Rhodocytin alpha chain \#1 & Ionic bond & ASA & $\overline{\text { BSA }}$ & $\Delta \mathbf{i G}$ \\
\hline GLN 23 & & 121.22 & $3.01 \mid$ & -0.01 \\
\hline THR 25 & & 22.63 & 0.37 | & \\
\hline GLU 28 & & 75.89 & $9.81 \|$ & \\
\hline ASP 100 & & 142.06 & $7.22 \mid$ & -0.12 \\
\hline HIS 102 & & 113.98 & $18.44 \|$ & -0.05 \\
\hline GLU 124 & & 74.46 & $21.29 \ddot{\| i}$ & 0.20 \\
\hline GLN 125 & & 78.37 & $23.20 i \|$ & -0.00 \\
\hline & & & & \\
\hline
\end{tabular}

\begin{tabular}{|c|c|c|c|c|}
\hline CLEC-2 & Ionic bond & ASA & BSA & $\Delta \mathbf{i G}$ \\
\hline ASP 188 & & 138.86 & $\begin{array}{l}6.93 \mid \\
\end{array}$ & -0.03 \\
\hline LYS 190 & & 135.33 & 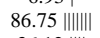 & -0.38 \\
\hline ASN 192 & & 110.81 & 36.12|||||| & -0.02 \\
\hline PHE 207 & & 59.76 & 31.74|||||||| $\mid$ & 0.51 \\
\hline ASN 210 & & 66.39 & $2.72 \mid$ & 0.00 \\
\hline
\end{tabular}

\begin{tabular}{|c|c|c|c|c|}
\hline & \multicolumn{2}{|c|}{ Rhodocytin beta chain \#1 } & \multicolumn{2}{|c|}{ CLEC-2 } \\
\hline $\begin{array}{c}\text { Number of atoms } \\
\text { interface } \\
\text { surface } \\
\text { total }\end{array}$ & $\begin{array}{r}18 \\
638 \\
1035 \\
\end{array}$ & $\begin{array}{l}(1.7 \%) \\
(61.6 \%) \\
(100.0 \%)\end{array}$ & $\begin{array}{c}12 \\
1129 \\
2085\end{array}$ & $\begin{array}{l}(0.6 \%) \\
(54.1 \%) \\
(100.0 \%)\end{array}$ \\
\hline $\begin{array}{c}\text { Number of residues } \\
\text { interface } \\
\text { surface } \\
\text { total }\end{array}$ & $\begin{array}{c}8 \\
112 \\
123\end{array}$ & $\begin{array}{r}(6.5 \%) \\
(91.1 \%) \\
(100.0 \%)\end{array}$ & $\begin{array}{c}6 \\
222 \\
242\end{array}$ & $\begin{array}{l}(2.5 \%) \\
(91.7 \%) \\
(100.0 \%)\end{array}$ \\
\hline $\begin{array}{l}\text { Solvent-accessible area, } \tilde{\AA}^{2} \\
\text { interface } \\
\text { total }\end{array}$ & $\begin{array}{r}130.6 \\
7933.1\end{array}$ & $\begin{array}{c}(1.6 \%) \\
(100.0 \%)\end{array}$ & $\begin{array}{c}154.2 \\
12294.5\end{array}$ & $\begin{array}{l}(1.3 \%) \\
(100.0 \%)\end{array}$ \\
\hline $\begin{array}{l}\text { Solvation energy, kcal/mol } \\
\text { isolated structure } \\
\text { gain at complexation } \\
\text { average gain } \\
P \text {-value }\end{array}$ & $\begin{array}{l}-107.9 \\
-0.2 \\
-0.5 \\
0.609\end{array}$ & $\begin{array}{l}(100.0 \%) \\
(0.2 \%) \\
(0.5 \%)\end{array}$ & $\begin{array}{l}-222.4 \\
1.7 \\
-0.1 \\
0.935\end{array}$ & $\begin{array}{l}(100.0 \%) \\
(0.0 \%) \\
(0.0 \%)\end{array}$ \\
\hline
\end{tabular}

\begin{tabular}{|c|c|c|c|c|}
\hline Rhodocytin alpha chain \#2 & Ionic bond & ASA & BSA & $\Delta \mathbf{i G}$ \\
\hline GLN 23 & $\mathrm{H}$ & 120.27 & $74.93 \mathrm{IIII \| I|}$ & -0.33 \\
\hline GLU 28 & & 81.78 & $1.71 \mid$ & -0.02 \\
\hline ASP 66 & & 125.72 & 28.70\|\| & 0.14 \\
\hline GLU 67 & & 18.44 & 1.15 & 0.02 \\
\hline ASP 68 & HS & 85.58 & 75.58|||||||||| & -0.77 \\
\hline TYR 69 & & 37.03 & $3.53 \mid$ & 0.05 \\
\hline ASP 100 & HS & 142.47 & 47.16|||| $\mid$ & -0.02 \\
\hline HIS 102 & & 113.24 & $7.72 \mid$ & -0.29 \\
\hline LYS 111 & $\mathrm{H}$ & 91.53 & $63.63\|\mid\|\|\| \|$ & -0.03 \\
\hline LEU 112 & & 155.55 & $127.31|\||\||\|\|\|\| \||$ & 1.55 \\
\hline GLY 114 & & 47.05 & $5.54 \|$ & 0.06 \\
\hline GLU 124 & & 72.61 & $2.09 \mid$ & -0.02 \\
\hline GLN 125 & & 72.41 & 55.90\|\|\|\|\|\| & 0.02 \\
\hline MET 126 & & 94.24 & 79.53 - & 1.92 \\
\hline HIS 127 & & 19.00 & 12.81\|\|\|\|$\| \mid$ & 0.20 \\
\hline Rhodocytin beta chain & Ionic bond & ASA & $\begin{array}{l}\text { BSA } \\
\end{array}$ & $\Delta \mathbf{i G}$ \\
\hline ASN 22 & & 69.56 & 20.99|||| & -0.00 \\
\hline ARG 100 & & 96.44 & $12.38 \|$ & -0.16 \\
\hline GLU 105 & HS & 81.89 & 22.63|| $\mid$ & -0.07 \\
\hline LEU 107 & & 47.78 & 10.71\|\| & 0.17 \\
\hline ASP 110 & & 76.19 & $0.24 \mid$ & -0.00 \\
\hline SER 112 & $\mathrm{H}$ & 79.62 & 35.64|||||| & -0.17 \\
\hline SER 113 & & 39.33 & $5.85 \|$ & 0.09 \\
\hline THR 114 & & 83.92 & $22.18 \|$ & 0.35 \\
\hline
\end{tabular}

\begin{tabular}{|c|c|c|c|c|}
\hline CLEC-2 & Ionic bond & ASA & BSA & $\Delta \mathrm{iG}$ \\
\hline ASN 120 & \multirow{6}{*}{ HS } & 97.19 & $3.68 \mid$ & 0.00 \\
\hline LYS 150 & & 76.82 & $4.77 \mid$ & 0.03 \\
\hline HIS 154 & & 42.91 & 12.38|||| & -0.24 \\
\hline LEU 155 & & 46.25 & $\begin{array}{l}4.86 \| \\
\|\end{array}$ & 0.08 \\
\hline ILE 156 & & 42.53 & 22.40||$\||\||$ & 0.02 \\
\hline GLU 187 & & $\begin{array}{l}57.90 \\
38.02\end{array}$ & $\begin{array}{r}1.47 \mid \\
1089\|\|\end{array}$ & -0.02 \\
\hline $\begin{array}{l}\text { TYR } 197 \\
\text { HIS } 199\end{array}$ & \multirow{6}{*}{$\mathrm{H}$} & $\begin{array}{l}38.02 \\
51.26\end{array}$ & $\begin{array}{c}10.89\|\| \| \\
47.31\|\| \|\end{array}$ & $\begin{array}{l}-0.11 \\
-0.90\end{array}$ \\
\hline ASN 200 & & 79.07 & $56.83\|\mid\|\|\|\|\|$ & -0.09 \\
\hline LYS 202 & & 95.17 & $47.42 \mathrm{II \| \| "}$ & -0.42 \\
\hline HIS 204 & & 43.12 & 29.08 |iाi|||| & 0.07 \\
\hline GLU 209 & & 125.40 & $0.61 \mid$ & -0.01 \\
\hline ASN 210 & & 66.39 & $8.19 \|$ & 0.01 \\
\hline $\begin{array}{l}\text { LYS } 211 \\
\text { HIS } 21\end{array}$ & \multirow{6}{*}{ HS } & $\begin{array}{l}122.09 \\
3227\end{array}$ & $33.70 \mathrm{i \|}$ & -0.14 \\
\hline ARG 118 & & $\begin{array}{r}52.27 \\
100.93\end{array}$ & 45.82|||||||| & $\begin{array}{l}0.19 \\
-0.76\end{array}$ \\
\hline ASN 120 & & 102.89 & 66.75|||||||||| $\mid$ & 0.09 \\
\hline LEU 155 & & 42.73 & $22.76 \mid \mathrm{IIIU \|}$ & 0.36 \\
\hline ILE 156 & & 44.58 & 18.67||$i|||||| \mid$ & 0.05 \\
\hline LYS 211 & & 116.36 & $11.88 \|$ & -0.04 \\
\hline $\begin{array}{c}\text { HIS } 212 \\
\text { TYR } 213\end{array}$ & $\mathrm{H}$ & $\begin{array}{l}33.89 \\
60.85\end{array}$ & 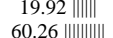 & $\begin{array}{l}-0.78 \\
-0.15\end{array}$ \\
\hline CLEC-2 & Ionic bond & $\overline{A S S A}$ & BSA & $\Delta \mathrm{iG}$ \\
\hline ASN 120 & \multirow{4}{*}{ HS } & 97.19 & $23.61 \|||$ & 0.02 \\
\hline LYS 211 & & 122.09 & 27.30|||| $\mid$ & -1.01 \\
\hline GLU 187 & & 54.63 & $19.30 \mid \underline{101}$ & -0.30 \\
\hline ASP 188 & & 135.67 & $6.75 \mid$ & -0.08 \\
\hline $\begin{array}{l}\text { LYS } 202 \\
\text { HIS } 204\end{array}$ & $\mathrm{H}$ & $\begin{array}{l}90.50 \\
4824\end{array}$ & 65.54|||||||||||| & -0.48 \\
\hline & & & & \\
\hline
\end{tabular}


Table 2. Contact information and interfacing residues involved in the model interaction of two copies of dimeric CLEC-2 with tetrameric rhodocytin. Key: $\mathrm{H}=$ residues making hydrogen bonds, $\mathrm{S}=$ residues making a salt bridge, ASA = accessible surface area $\left(\AA^{2}\right)$, BSA $=$ buried surface area $\left(\AA^{2}\right), \Delta \mathrm{iG}=$ solvation energy effect $(\mathrm{kcal} / \mathrm{mol}),\|\| \mid=$ buried area percentage, one bar per $10 \%$.

\begin{tabular}{|c|c|c|c|c|}
\hline & \multicolumn{2}{|c|}{ Rhodocytin alpha chain \#1 } & \multicolumn{2}{|c|}{$\begin{array}{l}\text { CLEC-2 } \\
\end{array}$} \\
\hline $\begin{array}{c}\text { Number of atoms } \\
\text { interface } \\
\text { surface } \\
\text { total } \\
\end{array}$ & $\begin{array}{c}19 \\
698 \\
1079\end{array}$ & $\begin{array}{l}(1.8 \%) \\
(64.7 \%) \\
(100.0 \%)\end{array}$ & $\begin{array}{c}14 \\
1091 \\
2029\end{array}$ & $\begin{array}{l}(0.7 \%) \\
(53.8 \%) \\
(100.0 \%)\end{array}$ \\
\hline $\begin{array}{c}\text { Number of residues } \\
\text { interface } \\
\text { surface } \\
\text { total } \\
\end{array}$ & $\begin{array}{l}11 \\
123 \\
132 \\
\end{array}$ & $\begin{array}{l}(8.3 \%) \\
(93.2 \%) \\
(100.0 \%)\end{array}$ & $\begin{array}{c}8 \\
219 \\
241\end{array}$ & $\begin{array}{l}(3.3 \%) \\
(90.9 \%) \\
(100.0 \%)\end{array}$ \\
\hline $\begin{array}{l}\text { Solvent-accessible area, } \tilde{\AA}^{2} \\
\text { interface } \\
\text { total }\end{array}$ & $\begin{array}{c}86.1 \\
8163.2\end{array}$ & $\begin{array}{c}(1.1 \%) \\
(100.0 \%)\end{array}$ & $\begin{array}{c}95.7 \\
11998.6\end{array}$ & $\begin{array}{c}(0.8 \%) \\
(100.0 \%)\end{array}$ \\
\hline $\begin{array}{l}\text { Solvation energy, kcal/mol } \\
\text { siolated structure } \\
\text { gain at complexation } \\
\text { average gain } \\
P \text {-value }\end{array}$ & $\begin{array}{l}-102.9 \\
-0.2 \\
-0.5 \\
0.590 \\
\end{array}$ & $\begin{array}{l}(100.0 \%) \\
(0.2 \%) \\
(0.4 \%)\end{array}$ & $\begin{array}{c}-240.7 \\
0.7 \\
0.0 \\
0.737\end{array}$ & $\begin{array}{l}(100.0 \%) \\
(-0.3 \%) \\
(-0.0 \%)\end{array}$ \\
\hline & \multicolumn{2}{|c|}{ Rhodocytin alpha chain \#2 } & \multicolumn{2}{|c|}{ CLEC-2 } \\
\hline $\begin{array}{l}\text { Number of atoms } \\
\text { interface } \\
\text { surface } \\
\text { total }\end{array}$ & $\begin{array}{c}51 \\
699 \\
1079\end{array}$ & $\begin{array}{l}(4.7 \%) \\
(64.8 \%) \\
(100.0 \%)\end{array}$ & $\begin{array}{c}53 \\
1085 \\
2029\end{array}$ & $\begin{array}{l}(2.6 \%) \\
(53.5 \%) \\
(100.0 \%)\end{array}$ \\
\hline $\begin{array}{c}\text { Number of residues } \\
\text { interface } \\
\text { surface } \\
\text { total }\end{array}$ & $\begin{array}{l}14 \\
123 \\
132\end{array}$ & $\begin{array}{l}(10.6 \%) \\
(93.2 \%) \\
(100.0 \%)\end{array}$ & $\begin{array}{l}19 \\
220 \\
241\end{array}$ & $\begin{array}{l}(7.9 \%) \\
(91.3 \%) \\
(100.0 \%)\end{array}$ \\
\hline $\begin{array}{l}\text { Solvent-accessible area, } \tilde{\AA}^{2} \\
\text { interface } \\
\text { total }\end{array}$ & $\begin{array}{r}562.3 \\
8165.3\end{array}$ & $\begin{array}{c}(6.9 \%) \\
(100.0 \%)\end{array}$ & $\begin{array}{c}459.1 \\
12027.2\end{array}$ & $\begin{array}{c}(3.8 \%) \\
(100.0 \%)\end{array}$ \\
\hline $\begin{array}{l}\text { Solvation energy, kcal/mol } \\
\text { isolated structure } \\
\text { gain at complexation } \\
\text { average gain } \\
P \text {-value }\end{array}$ & $\begin{array}{l}-103.6 \\
-0.6 \\
-1.2 \\
0.638\end{array}$ & $\begin{array}{l}(100.0 \%) \\
(0.6 \%) \\
(1.2 \%)\end{array}$ & $\begin{array}{c}-240.8 \\
-0.5 \\
0.2 \\
0.406\end{array}$ & $\begin{array}{c}(100.0 \%) \\
(0.2 \%) \\
(-0.1 \%)\end{array}$ \\
\hline
\end{tabular}

\begin{tabular}{|c|c|c|c|c|}
\hline Rhodocytin alpha chain \#1 & Ionic bond & ASA & BSA & $\Delta \mathbf{i G}$ \\
\hline GLN 23 & \multirow{11}{*}{ HS } & 108.13 & 1.221 & -0.02 \\
\hline GLU 67 & & 14.14 & $0.49 \mid$ & -0.01 \\
\hline ASP 68 & & 81.41 & $35.61 \mathrm{IIIII}$ & -0.14 \\
\hline TYR 69 & & 36.42 & $7.12 \|||$ & 0.11 \\
\hline ASP 100 & & 141.21 & 0.50 & 0.01 \\
\hline LEU 112 & & 147.96 & $15.33 \|$ & 0.06 \\
\hline THR 113 & & 45.14 & 4.02 & 0.04 \\
\hline TYR 121 & & 35.14 & 2.70 & -0.03 \\
\hline TYR 122 & & 69.39 & 2.21 & -0.03 \\
\hline GLN 125 & & 64.01 & $1.17 \mid$ & -0.01 \\
\hline HIS 127 & & 18.53 & 15.77\|\|\|\|$\|$ & 0.25 \\
\hline
\end{tabular}

\begin{tabular}{|c|c|c|c|c|}
\hline $\begin{array}{l}\text { CLEC-2 } \\
\end{array}$ & Ionic bond & ASA & BSA & $\Delta \mathbf{i G}$ \\
\hline ARG 143 & $\mathrm{HS}$ & 161.22 & 52.59|||| $\mid$ & -0.74 \\
\hline ASN 144 & & 82.06 & $\left.1.75\right|^{\prime \prime}$ & -0.02 \\
\hline GLU 147 & & 55.82 & 16.53|| $\mid$ & -0.14 \\
\hline LYS 150 & & 79.33 & $\left.0.26\right|^{\prime \prime}$ & -0.01 \\
\hline GLU 184 & & 139.38 & $1.23 \mid$ & -0.01 \\
\hline $\begin{array}{l}\text { ASP } 188^{4} \\
\text { A }\end{array}$ & & $\begin{array}{l}139.39 \\
\end{array}$ & 0.49 & -0.01 \\
\hline LYS 202 & & 96.42 & $\begin{array}{l}0.47 \mid \\
2.31\end{array}$ & 0.03 \\
\hline HIS 119 & & 80.14 & 20.51|| $\mid$ & 0.23 \\
\hline
\end{tabular}

\begin{tabular}{|c|c|c|c|c|}
\hline \multirow[b]{2}{*}{ 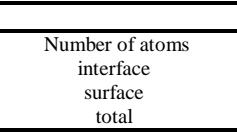 } & \multicolumn{2}{|c|}{ Rhodocytin beta chain \#1 } & \multicolumn{2}{|c|}{ CLEC-2 } \\
\hline & $\begin{array}{c}44 \\
626 \\
1006\end{array}$ & $\begin{array}{l}(4.4 \%) \\
(62.2 \%) \\
(100.0 \%)\end{array}$ & $\begin{array}{c}45 \\
1085 \\
2029\end{array}$ & $\begin{array}{l}(2.2 \%) \\
(53.5 \%) \\
(100.0 \%)\end{array}$ \\
\hline $\begin{array}{c}\text { Number of residues } \\
\text { interface } \\
\text { surface } \\
\text { total } \\
\end{array}$ & $\begin{array}{l}15 \\
111 \\
122\end{array}$ & $\begin{array}{l}(12.3 \%) \\
(91.0 \%) \\
(100.0 \%)\end{array}$ & $\begin{array}{l}16 \\
220 \\
241\end{array}$ & $\begin{array}{l}(6.6 \%) \\
(91.3 \%) \\
(100.0 \%)\end{array}$ \\
\hline $\begin{array}{l}\text { Solvent-accessible area, } \AA^{2} \\
\text { interface } \\
\text { total }\end{array}$ & $\begin{array}{r}390.7 \\
7813.8\end{array}$ & $\begin{array}{c}(5.0 \%) \\
(100.0 \%)\end{array}$ & $\begin{array}{c}411.1 \\
12027.2\end{array}$ & $\begin{array}{c}(3.4 \%) \\
(100.0 \%)\end{array}$ \\
\hline $\begin{array}{l}\text { Solvation energy, kcal/mol } \\
\text { isolated structure } \\
\text { gain at complexation } \\
\text { average gain } \\
P \text {-value }\end{array}$ & $\begin{array}{l}-111.3 \\
-2.2 \\
-0.8 \\
0.290\end{array}$ & $\begin{array}{c}(100.0 \%) \\
(1.9 \%) \\
(0.8 \%)\end{array}$ & $\begin{array}{c}-240.8 \\
1.8 \\
0.1 \\
0.799\end{array}$ & $\begin{array}{l}(100.0 \%) \\
(-0.7 \%) \\
(-0.1 \%)\end{array}$ \\
\hline
\end{tabular}

\begin{tabular}{|c|c|c|c|c|}
\hline Rhodocytin alpha chain \#2 & Ionic bond & ASA & 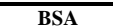 & $\Delta \mathbf{A i G}$ \\
\hline ASP 66 & $\mathrm{HS}$ & 130.36 & 79.17|||||| $\mid$ & -0.03 \\
\hline ASP 68 & & 81.94 & (71.50 & -0.55 \\
\hline TYR 69 & & 36.31 & 27.26|||||||| $\mid$ & -0.04 \\
\hline LYS 104 & & 93.33 & $36.30|\|| \mid$ & -1.07 \\
\hline GLU 110 & & 23.39 & $6.91 \|||$ & -0.06 \\
\hline LYS 111 & & 93.46 & $64.69 \mid\|\|\|\| \|$ & 0.62 \\
\hline LEU 112 & & 147.63 & 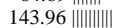 & 1.57 \\
\hline THR 113 & & 44.93 & 9.95|| $\mid$ & 0.07 \\
\hline GLY 114 & & 46.88 & 2.48 & -0.03 \\
\hline LYS 117 & & $\begin{array}{l}40.00 \\
141.41\end{array}$ & $26.11 \|$ & 0.42 \\
\hline TYR 121 & & 35.30 & 25.21\|\|\|\|$\|$ & -0.13 \\
\hline TYR 122 & & 68.95 & 35.37|||||| $\mid$ & 0.07 \\
\hline GLN 125 & $\mathrm{H}$ & 63.99 & 30.94\|\|\|\| & -0.33 \\
\hline & & & & \\
\hline Rhodocytin beta chain \#1 & Ionic bond & ASA & BSA & $\Delta \mathbf{i G}$ \\
\hline PRO 20 & \multirow[t]{7}{*}{ Howe word } & 80.18 & $7.37 \mid$ & 0.12 \\
\hline LEU 63 & & $\begin{array}{l}80.18 \\
30.42\end{array}$ & $8.55 \|$ & $\begin{array}{l}0.12 \\
0.14\end{array}$ \\
\hline $\begin{array}{l}\text { LEU } 93 \\
\text { GLN } 91\end{array}$ & & $\begin{array}{r}00.42 \\
119.63\end{array}$ & 31.40\|\| & $\begin{array}{l}0.14 \\
-0.47\end{array}$ \\
\hline GLU 92 & & 158.11 & 54.64\|\|\|\| & -0.10 \\
\hline GLN 93 & & 105.23 & $12.72 \|$ & 0.20 \\
\hline $\begin{array}{l}\text { SLN } 93 \\
\text { SER } 94\end{array}$ & & $\begin{array}{l}10.239 \\
30.87\end{array}$ & $\begin{array}{l}12.12 \pi \\
0.24 \mid\end{array}$ & $\begin{array}{l}0.20 \\
-0.00\end{array}$ \\
\hline GLU 95 & & 50.04 & 24.82|||||| & -0.19 \\
\hline ARG 100 & \multirow[b]{2}{*}{$\mathrm{H}$} & 98.07 & $\begin{array}{l}2.82 \mathrm{IIII} \\
3.35\end{array}$ & $\begin{array}{l}-0.19 \\
-0.12\end{array}$ \\
\hline ASN 108 & & 74.11 & 14.85|| $\mid$ & -0.22 \\
\hline $\begin{array}{l}\text { ASN } 108 \\
\text { MET } 109\end{array}$ & \multirow{6}{*}{$\mathrm{H}$} & $\begin{array}{l}74.11 \\
53.08\end{array}$ & $\begin{array}{l}14.85\|\|\|\| \| \\
44.57|| \|||\end{array}$ & $\begin{array}{l}-0.22 \\
1.42\end{array}$ \\
\hline ASP 110 & & 75.54 & $\begin{array}{l}64.97|||||||||||| \\
6 \mid\end{array}$ & $\begin{array}{l}1.42 \\
0.15\end{array}$ \\
\hline SER 112 & & 83.61 & $\begin{array}{l}-24.97|||||||||| \mid \\
44.24|||| \mid\end{array}$ & $\begin{array}{l}.13 \\
-0.13\end{array}$ \\
\hline SER 113 & & $\begin{array}{l}83.61 \\
37.64\end{array}$ & $\begin{array}{l}45.24\|\|\|\|\|\| \| \\
35.97\|\|\end{array}$ & 0.58 \\
\hline THR 114 & & 82.96 & 38.52|||||| $\mid$ & 0.60 \\
\hline CYS 115 & & $\begin{array}{l}82.14 \\
10.14\end{array}$ & 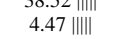 & 0.18 \\
\hline
\end{tabular}

\begin{tabular}{|c|c|c|c|c|}
\hline CLEC-2 & Ionic bond & ASA & BSA & $\Delta \mathbf{i} \mathbf{G}$ \\
\hline ASN 120 & \multirow{19}{*}{$\mathrm{HS}$} & $\begin{array}{l}78.74 \\
\end{array}$ & $8.01 \|$ & -0.09 \\
\hline HIS 154 & & 47.02 & $20.87\|\mid\| \|$ & -0.20 \\
\hline LEU 155 & & 35.70 & 29.71|||||||||||| & 0.48 \\
\hline ILE 156 & & 53.72 & 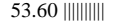 & 0.73 \\
\hline GLU 187 & & 56.68 & $10.36 \|$ & -0.12 \\
\hline TYR 197 & & 32.06 & 19.76||||$|w|$ & -0.19 \\
\hline & & 50.16 & 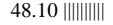 & 0.23 \\
\hline ASN 200 & & 79.01 & 17.04|| $\mid$ & -0.12 \\
\hline LYS 202 & & 98.88 & 29.20|||| $\mid$ & -0.57 \\
\hline HIS 204 & & 50.56 & 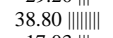 & 0.13 \\
\hline PHE 207 & & 75.23 & 17.03|||| & 0.27 \\
\hline GLU 209 & & 126.27 & $3.80 \mid$ & -0.04 \\
\hline ASN 210 & & 58.58 & 45.69|||||||||||| & -0.24 \\
\hline LYS 211 & & 115.90 & $44.00 \mathrm{\| \| ||-1}$ & -0.06 \\
\hline HIS 212 & & 35.13 & 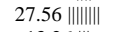 & 0.15 \\
\hline TYR 213 & & 66.22 & $13.26 \|||$ & 0.21 \\
\hline HIS 154 & & 29.40 & 6.68|| $\mid$ & -0.08 \\
\hline HIS 199 & & 52.25 & 16.26|||| $\mid$ & 0.15 \\
\hline ASN 200 & & 73.94 & $9.33 \|$ & -0.16 \\
\hline CLEC-2 & Ionic bond & ASA & $\begin{array}{ll}\text { BSA } \\
\text { Bats }\end{array}$ & $\Delta \mathrm{iG}$ \\
\hline ARG 118 & \multirow{16}{*}{ HS } & 61.27 & $6.80 \|$ & -0.01 \\
\hline HIS 119 & & 78.21 & $35.49\||\||\||$ & $\begin{array}{l}-0.68 \\
\end{array}$ \\
\hline ASN 120 & & 78.74 & 50.66||$|W| W \mid$ & -0.21 \\
\hline LEU 121 & & 32.06 & 29.72 ||||||||||||||| & 0.48 \\
\hline THR 122 & & & 12.79|||||| & 0.20 \\
\hline GLU 124 & & 105.69 & 3.56 & 0.06 \\
\hline GLU 125 & & 90.53 & 64.70\|\|\|\|$\|$ & -0.51 \\
\hline GLN 128 & & 94.77 & $6.26 \mid$ & 0.10 \\
\hline TYR 129 & & 54.87 & 16.90|||||| & 0.27 \\
\hline ASP 132 & & 99.68 & 21.00\|\| & -0.17 \\
\hline LEU 155 & & 35.70 & $5.35 \|$ & 0.09 \\
\hline LYS 211 & & 115.90 & 71.91|||||||||| & -1.17 \\
\hline TYR 213 & & 66.22 & $33.75 \mathrm{\| \| \| \| \|}$ & -0.29 \\
\hline LYS 150 & & 75.88 & $2.85 \mid$ & 0.05 \\
\hline HIS 154 & & 29.40 & 1.51 & 0.02 \\
\hline ASN 200 & & 73.94 & $47.86 \mathrm{IIUW}$ & 0.02 \\
\hline
\end{tabular}


Figure 1. Representation of an interface between CLEC-2 (white) and a beta subunit (pink) of the rhodocytin tetramer. Sidechains of interacting residues (N120 and K211 on CLEC-2, and E95 and D110 on the beta subunit of rhodocytin) are represented as balls and sticks. Predicted hydrogen bonds are represented by broken dotted blue lines.

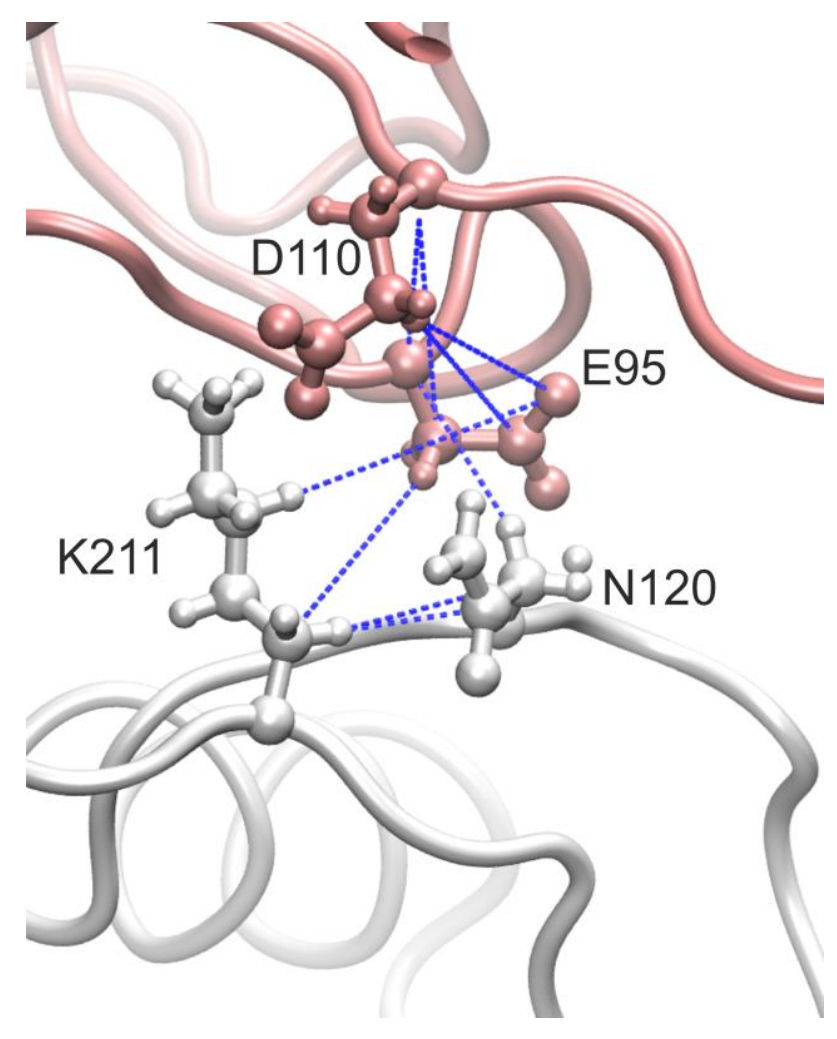

\subsection{Dynamic Flexibility of the Rhodocytin-CLEC-2 Complexes}

Molecular dynamics analyses were undertaken to investigate the potential flexibility of the two possible rhodocytin-CLEC-2 interaction models (Figures 2, 3). With only a single dimer of CLEC-2 interacting with tetrameric rhodocytin, the predicted motions are dominated by the alpha and beta subunits of rhodocytin and the motions of CLEC-2 appear to play a relatively minor part in the overall flexibility of the complex (Figure 2). Interestingly, in the model with two copies of dimeric CLEC-2 bound to rhodocytin, a much greater contribution is made by the CLEC-2 dimers, and the beta subunits of rhodocytin, to the global flexibility of the complex (Figure 3). In the molecular dynamics simulations for this model, the CLEC-2 dimers appear to wrap around the grooves presented on rhodocytin, and the beta subunits move in a complementary fashion so as to maximise the exposure and accessibility of the two binding grooves to CLEC-2 (Figure 3). Thus, in this interaction mode with two CLEC-2 molecules, the alpha subunits of rhodocytin make a relatively more minor contribution to the possible dynamic motions of the complex (Figure 3 ). 
Figure 2. Dynamic analyses of a model of the interaction of dimeric CLEC-2 (white) with tetrameric rhodocytin. The rhodocytin $\alpha$ - and $\beta$-chains are coloured yellow and pink, respectively. The porcupine plots represent the principal mode of conformational variability of the $\mathrm{C} \alpha$ atoms calculated from a CONCOORD ensemble using the model of the rhodocytin-CLEC-2 interaction based on the crystal structure of rhodocytin, and a model of the dimeric structure of CLEC-2. Blue cones represent the direction of each motion; the length of the cone is proportional to the amplitude of the motion. The top image represents a $90^{\circ}$ counter-clockwise rotation of the central image about the $\mathrm{X}$-axis. The bottom image represents a $90^{\circ}$ clockwise rotation of the top image about the Y-axis.
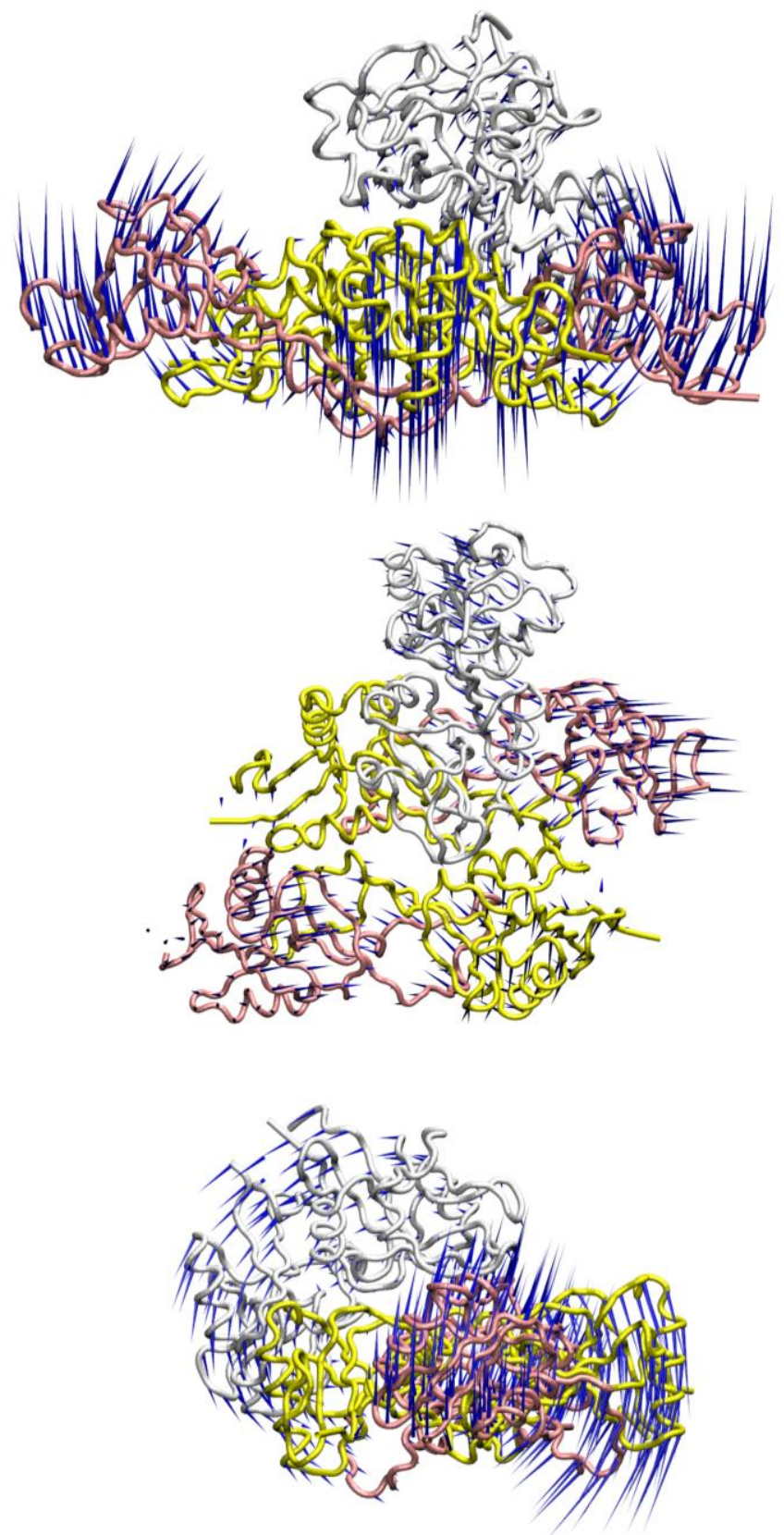
Figure 3. Dynamic analyses of a model of the interaction of two copies of dimeric CLEC-2 (white) with tetrameric rhodocytin. The rhodocytin $\alpha$ - and $\beta$-chains are coloured yellow and pink, respectively. The porcupine plots represent the principal mode of conformational variability of the $\mathrm{C} \alpha$ atoms calculated from a CONCOORD ensemble using the model of the rhodocytin-CLEC-2 interaction based on the crystal structure of rhodocytin, and a model of the dimeric structure of CLEC-2. Blue cones represent the direction of each motion; the length of the cone is proportional to the amplitude of the motion. The top image represents a $90^{\circ}$ counter-clockwise rotation of the central image about the $\mathrm{X}$-axis. The bottom image represents a $90^{\circ}$ clockwise rotation of the top image about the Y-axis.

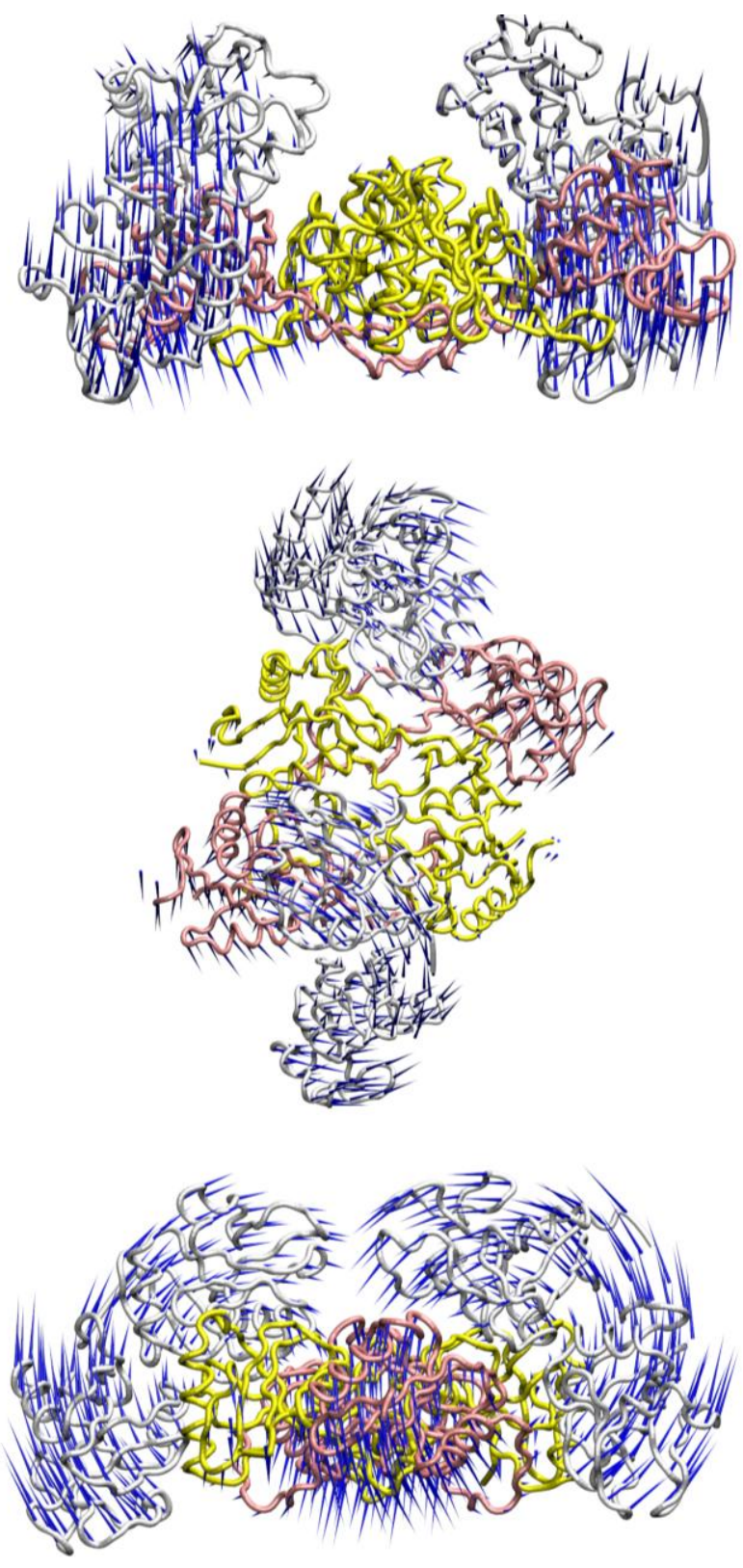

\section{Conclusions}

We have previously solved the crystal structures of both rhodocytin and CLEC-2, but there is no crystallographic structure of the rhodocytin-CLEC-2 complex. Using three dimensional structures of 
both rhodocytin and CLEC-2, we have generated models of the likely modes of interaction of the venom protein and its receptor on platelets and have investigated and analysed the computational models of the interaction which we have generated [30,32,33]. Using a set of analytical algorithms and approaches, we have assessed the properties of the interfacing surfaces and the contribution made to the interaction by specific intermolecular contacts, including salt bridges and hydrogen bonds. In addition to this, we have evaluated the potential flexibility of these model complexes. The model wherein two molecules of CLEC-2 associate with tetrameric rhodocytin provides a more plausible model in terms of the composite effects related to the number of interfacing residues, the nature of their interactions and the predicted solvation energy effects. Further, it is of potential significance that the predicted dynamic motions of this complex are suggestive of a mechanism whereby this interaction might cluster the receptors on the platelet surface, which could have implications for signaling. Overall, the work presented indicates that a plausible mode of binding is that of one non-disulfide linked $(\alpha \beta)_{2}$ tetramer of rhodocytin with two dimers of CLEC-2. This analysis will be of value in the development of further studies to characterise the interaction further with a view to developing therapeutic approaches to disrupt the rhodocytin-CLEC-2 interaction on the platelet surface.

\section{Acknowledgments}

This work was funded by the British Heart Foundation. AAW is a Sir Henry Wellcome Postdoctoral Fellow. We are grateful to Steve Watson for helpful discussions.

\section{Conflict of Interest}

The authors declare no conflict of interest.

\section{References}

1. Pirkle, H.; Markland, F.S., Jr. Hemostasis and Animal Venoms; Dekker: New York, NY, USA, 1987.

2. Clemetson, K.J.; Lu, Q.; Clemetson, J.M. Snake venom proteins affecting platelets and their applications to anti-thrombotic research. Curr. Pharm. Des. 2007, 13, 2887-2892.

3. Clemetson, K.J.; Lu, Q.; Clemetson, J.M. Snake C-type lectin-like proteins and platelet receptors. Pathophysiol. Haemost. Thromb. 2005, 34, 150-155.

4. Ruggeri, Z.M. Platelets in atherothrombosis. Nat. Med. 2002, 8, 1227-1234.

5. Lusis, A.J. Atherosclerosis. Nature 2000, 407, 233-241.

6. Ho, M.; Warrell, D.A.; Looareesuwan, S.; Phillips, R.E.; Chanthavanich, P.; Karbwang, J.; Supanaranond, W.; Viravan, C.; Hutton, R.A.; Vejcho, S. Clinical significance of venom antigen levels in patients envenomed by the malayan pit viper (Calloselasma rhodostoma). Am. J. Trop. Med. Hyg. 1986, 35, 579-587.

7. Huang, T.F.; Liu, C.Z.; Yang, S.H. Aggretin, a novel platelet-aggregation inducer from snake (Calloselasma rhodostoma) venom, activates phospholipase c by acting as a glycoprotein ia/iia agonist. Biochem. J. 1995, 309, 1021-1027. 
8. Shin, Y.; Morita, T. Rhodocytin, a functional novel platelet agonist belonging to the heterodimeric c-type lectin family, induces platelet aggregation independently of glycoprotein ib. Biochem. Biophys. Res. Commun. 1998, 245, 741-745.

9. Chung, C.H.; Au, L.C.; Huang, T.F. Molecular cloning and sequence analysis of aggretin, a collagen-like platelet aggregation inducer. Biochem. Biophys. Res. Commun. 1999, 263, 723-727.

10. Suzuki-Inoue, K.; Fuller, G.L.; Garcia, A.; Eble, J.A.; Pohlmann, S.; Inoue, O.; Gartner, T.K.; Hughan, S.C.; Pearce, A.C.; Laing, G.D.; et al. A novel Syk-dependent mechanism of platelet activation by the C-type lectin receptor CLEC-2. Blood 2006, 107, 542-549.

11. Fuller, G.L.; Williams, J.A.; Tomlinson, M.G.; Eble, J.A.; Hanna, S.L.; Pohlmann, S.; Suzuki-Inoue, K.; Ozaki, Y.; Watson, S.P.; Pearce, A.C. The C-type lectin receptors CLEC-2 and Dectin-1, but not DC-SIGN, signal via a novel YXXL-dependent signaling cascade. J. Biol. Chem. 2007, 282, 12397-12409.

12. O'Callaghan, C.A. Thrombomodulation via CLEC-2 targeting. Curr. Opin. Pharmacol. 2009, 9, 90-95.

13. O’Callaghan, C.A.; Tormo, J.; Willcox, B.E.; Braud, V.M.; Jakobsen, B.K.; Stuart, D.I.; McMichael, A.J.; Bell, J.I.; Jones, E.Y. Structural features impose tight peptide binding specificity in the non-classical MHC molecule HLA-E. Mol. Cell 1998, 1, 531-541.

14. Braud, V.; Allan, D.S.J.; O’Callaghan, C.A.; Ogg, G.S.; Lazetic, S.; Young, N.T.; Bell, J.I.; Phillips, J.H.; Lanier, L.L.; McMichael, A.J. HLA-E binds to natural killer cell receptors CD94/NKG2A, B and C. Nature 1998, 391, 795-799.

15. O’Callaghan, C.A.; Cerwenka, A.; Willcox, B.E.; Lanier, L.L.; Bjorkman, P.J. Molecular competition for NKG2D: H60 and RAE1 compete unequally for NKG2D with dominance of H60. Immunity 2001, 15, 201-211.

16. Christou, C.M.; Pearce, A.C.; Watson, A.A.; Mistry, A.R.; Pollitt, A.Y.; Fenton-May, A.E.; Johnson, L.A.; Jackson, D.G.; Watson, S.P.; O’Callaghan, C.A. Renal cells activate the platelet receptor CLEC-2 through podoplanin. Biochem. J. 2008, 411, 133-140.

17. O'Callaghan, C.A.; Jones, E.Y. Structural and energetic aspects of multispecific immune recognition by NKG2D. Structure (Camb.) 2003, 11, 360-361.

18. Cueni, L.N.; Chen, L.; Zhang, H.; Marino, D.; Huggenberger, R.; Alitalo, A.; Bianchi, R.; Detmar, M. Podoplanin-fc reduces lymphatic vessel formation in vitro and in vivo and causes disseminated intravascular coagulation when transgenically expressed in the skin. Blood 2010, 116, 4376-4384.

19. Suzuki-Inoue, K.; Kato, Y.; Inoue, O.; Kaneko, M.K.; Mishima, K.; Yatomi, Y.; Narimatsu, H.; Ozaki, Y. Involvement of the snake toxin receptor CLEC-2 in podoplanin-mediated platelet activation by cancer cells. J. Biol. Chem. 2007, 282, 25993-26001.

20. Martin-Villar, E.; Scholl, F.G.; Gamallo, C.; Yurrita, M.M.; Munoz-Guerra, M.; Cruces, J.; Quintanilla, M. Characterization of human pa2.26 antigen (t1alpha-2, podoplanin), a small membrane mucin induced in oral squamous cell carcinomas. Int. J. Cancer 2005, 113, 899-910.

21. Uhrin, P.; Zaujec, J.; Breuss, J.M.; Olcaydu, D.; Chrenek, P.; Stockinger, H.; Fuertbauer, E.; Moser, M.; Haiko, P.; Fassler, R.; et al. Novel function for blood platelets and podoplanin in developmental separation of blood and lymphatic circulation. Blood 2010, 115, 3997-4005. 
22. Schacht, V.; Ramirez, M.I.; Hong, Y.K.; Hirakawa, S.; Feng, D.; Harvey, N.; Williams, M.; Dvorak, A.M.; Dvorak, H.F.; Oliver, G.; et al. T1alpha/podoplanin deficiency disrupts normal lymphatic vasculature formation and causes lymphedema. EMBO J. 2003, 22, 3546-3556.

23. Mahtab, E.A.; Vicente-Steijn, R.; Hahurij, N.D.; Jongbloed, M.R.; Wisse, L.J.; DeRuiter, M.C.; Uhrin, P.; Zaujec, J.; Binder, B.R.; Schalij, M.J.; et al. Podoplanin deficient mice show a rhoa-related hypoplasia of the sinus venosus myocardium including the sinoatrial node. Dev. Dyn. 2009, 238, 183-193.

24. Douglas, Y.L.; Mahtab, E.A.; Jongbloed, M.R.; Uhrin, P.; Zaujec, J.; Binder, B.R.; Schalij, M.J.; Poelmann, R.E.; Deruiter, M.C.; Gittenberger-de Groot, A.C. Pulmonary vein, dorsal atrial wall and atrial septum abnormalities in podoplanin knockout mice with disturbed posterior heart field contribution. Pediatr. Res. 2009, 65, 27-32.

25. Hughes, C.E.; Navarro-Núñez, L.; Finney, B.A.; Mourão-Sá, D.; Pollitt, A.Y.; Watson, S.P. CLEC-2 is not required for platelet aggregation at arteriolar shear. J. Thromb. Haemost. 2010, 8 , $2328-2332$.

26. Tang, T.; Li, L.; Tang, J.; Li, Y.; Lin, W.Y.; Martin, F.; Grant, D.; Solloway, M.; Parker, L.; Ye, W.; et al. A mouse knockout library for secreted and transmembrane proteins. Nat. Biotechnol. 2010, 28, 749-755.

27. Bertozzi, C.C.; Schmaier, A.A.; Mericko, P.; Hess, P.R.; Zou, Z.; Chen, M.; Chen, C.Y.; Xu, B.; Lu, M.M.; Zhou, D.; et al. Platelets regulate lymphatic vascular development through CLEC-2-SLP-76 signaling. Blood 2010, 116, 661-670.

28. Suzuki-Inoue, K.; Inoue, O.; Ding, G.; Nishimura, S.; Hokamura, K.; Eto, K.; Kashiwagi, H.; Tomiyama, Y.; Yatomi, Y.; Umemura, K.; et al. Essential in vivo roles of the C-type lectin receptor CLEC-2: Embryonic/neonatal lethality of CLEC-2-deficient mice by blood/lymphatic misconnections and impaired thrombus formation of CLEC-2-deficient platelets. J. Biol. Chem. 2010, 285, 24494-24507.

29. Chippaux, J.P. Snake-bites: Appraisal of the global situation. Bull. WHO 1998, 76, 515-524.

30. Watson, A.A.; Brown, J.; Harlos, K.; Eble, J.A.; Walter, T.S.; O’Callaghan, C.A. The crystal structure and mutational binding analysis of the extracellular domain of the platelet-activating receptor CLEC-2. J. Biol. Chem. 2007, 282, 3165-3172.

31. Watson, A.A.; O'Callaghan, C.A. Crystallization and X-ray diffraction analysis of human CLEC-2. Acta Crystallogr. Sect. F Struct. Biol. Cryst. Commun. 2005, 61, 1094-1096.

32. Watson, A.A.; Christou, C.M.; James, J.R.; Fenton-May, A.E.; Moncayo, G.E.; Mistry, A.R.; Davis, S.J.; Gilbert, R.J.; Chakera, A.; O'Callaghan, C.A. The platelet receptor CLEC-2 is active as a dimer. Biochemistry 2009, 48, 10988-10996.

33. Watson, A.A.; Eble, J.A.; O'Callaghan, C.A. Crystal structure of rhodocytin, a ligand for the platelet-activating receptor CLEC-2. Protein Sci. 2008, 17, 1611-1616.

34. Hughes, C.E.; Pollitt, A.Y.; Mori, J.; Eble, J.A.; Tomlinson, M.G.; Hartwig, J.H.; O'Callaghan, C.A.; Futterer, K.; Watson, S.P. CLEC-2 activates Syk through dimerization. Blood 2010, 115, 2947-2955.

35. Krissinel, E.; Henrick, K. Inference of macromolecular assemblies from crystalline state. J. Mol. Biol. 2007, 372, 774-797. 
36. Barrett, C.P.; Hall, B.A.; Noble, M.E. Dynamite: A simple way to gain insight into protein motions. Acta Crystallogr. D Biol. Crystallogr. 2004, 60, 2280-2287.

37. De Groot, B.L.; van Aalten, D.M.; Scheek, R.M.; Amadei, A.; Vriend, G.; Berendsen, H.J. Prediction of protein conformational freedom from distance constraints. Proteins 1997, 29, 240-251.

38. Humphrey, W.; Dalke, A.; Schulten, K. VMD: Visual molecular dynamics. J. Mol. Graph. 1996, 14, 33-38, 27-38.

(C) 2011 by the authors; licensee MDPI, Basel, Switzerland. This article is an open access article distributed under the terms and conditions of the Creative Commons Attribution license (http://creativecommons.org/licenses/by/3.0/). 